\title{
Joint Detection of Serum IgM/IgG Antibody Is an Important Key to Clinical Diagnosis of SARS-CoV-2 Infection
}

\author{
Fang Hu, Xiaoling Shang, Meizhou Chen (D), and Changliang Zhang \\ Department of Clinical Laboratory, Huangshi Central Hospital, \\ Edong Healthcare Group (Affiliated Hospital of Hubei Polytechnic University), No. 141 Tianjin Road, Huangshi City, \\ Hubei 435000, China \\ Correspondence should be addressed to Meizhou Chen; cmz245380374@163.com
}

Received 22 June 2020; Revised 25 August 2020; Accepted 9 September 2020; Published 24 September 2020

Academic Editor: José Ramón Blanco

Copyright ( $) 2020$ Fang Hu et al. This is an open access article distributed under the Creative Commons Attribution License, which permits unrestricted use, distribution, and reproduction in any medium, provided the original work is properly cited.

\begin{abstract}
Background. This study was aimed to investigate the application of SARS-CoV-2 IgM and IgG antibodies in diagnosis of COVID-19 infection. Method. This study enrolled a total of 178 patients at Huangshi Central Hospital from January to February 2020. Among them, 68 patients were SARS-CoV-2 infected, confirmed with nucleic acid test (NAT) and CT imaging. Nine patients were in the suspected group (NAT negative) with fever and other respiratory symptoms. 101 patients were in the control group with other diseases and negative to SARS-CoV-2 infection. After serum samples were collected, SARS-CoV-2 IgG and IgM antibodies were tested by chemiluminescence immunoassay (CLIA) for all patients. Results. The specificity of serum IgM and IgG antibodies to SARS-CoV-2 was 99.01\% (100/101) and 96.04\% (97/101), respectively, and the sensitivity was $88.24 \%(60 / 68)$ and $97.06 \%(66 / 68)$, respectively. The combined detection rate of SARS-CoV-2 IgM and IgG antibodies was 98.53\% (67/68). Conclusion. Combined detection of serum SARS-CoV-2 IgM and IgG antibodies had better sensitivity compared with single IgM or IgG antibody testing, which can be used as an important diagnostic tool for SARS-CoV-2 infection and a screening tool of potential SARS-CoV-2 carriers in clinics, hospitals, and accredited scientific laboratories.
\end{abstract}

\section{Introduction}

The novel coronavirus (SARS-CoV-2) is a new virus responsible for an outbreak of respiratory illness known as COVID-19, and now it is a global pandemic in more than 215 countries [1]. The current standard diagnostic method for diagnosis of COVID-19 is to detect the virus nucleic acid RT-PCR [2]. However, real-time PCR detection had some limitations, e.g., time-consuming, complicated operation with specialized equipment, and requiring special detection sites, which limit its application during COVID-19 outbreak [3]. Therefore, a simple, sensitive, and accurate test was urgently needed to identify SARS-CoV-2-infected patients in a COVID-19 outbreak area.

Based on the diagnosis experience of many clinical cases, the detection of novel coronavirus antibody can be used as an auxiliary diagnosis of novel coronavirus pneumonia $[4,5]$. After SARS-CoV-2 infection, the body's immune system can create immune response to fight against the virus and produce specific antibodies. In general virology, the immunoglobulin $\mathrm{M}$ (IgM) antibody, produced in the early period after the infection, can indicate the current infection or the recent infection. Immunoglobulin $\mathrm{G}$ (IgG) antibody is also an important antibody produced by the immune system, indicating that the disease is in the middle to late stage or presence of past infection. Therefore, the combined detection of IgM and IgG can be used not only in the early diagnosis of infectious diseases but also in the assessment of the stage of infection.

Chemiluminescence immunoassay (CLIA) has been developed as an effective combination of immunoassay and chemiluminescence system [6], and it has been used recently in SARS-CoV-2 diagnosis. In this study, we used a CLIA test product, which can detect IgM and IgG in human serum within 30 minutes. Our aim is to investigate the clinical value of CLIA for the diagnosis of SARS-CoV-2 infection. This 
CLIA method demonstrated good sensitivity and specificity in our study, which can be used not only in hospitals and accredited laboratories but also in airports, border ports, seaports, and train stations. This CLIA method has potential to be a powerful weapon against the COVID-19 pandemic.

\section{Materials and Methods}

2.1. Patients. This study enrolled a total of 178 patients who visited Huangshi Central Hospital in Hubei Province, China, between January and February 2020. The patients included 91 males $(51.1 \%)$ and 87 females $(48.9 \%)$ with a mean age of 54.3 years (ranging from 2 months to 94 years). Among them, the SARS-CoV-2 group had 68 patients, 36 males and 32 females (ranging from 30 years to 90 years); the suspected group had 9 patients, 7 males and 2 females (ranging from 2 months to 64 years); and the negative group had 101 patients, 48 males and 53 females (ranging from 2 years to 94 years). This study is in compliance with ICC clinical trial specifications and the Helsinki Declaration.

2.2. Serologic Tests. Serum was collected from all patients. Serum SARS-CoV-2 IgG and IgM were tested by CLIA kits and the iFlash 3000 fully automated CLIA analyzer obtained from Shenzhen YHLO Biotech Co., Ltd (China). In brief, serum was separated by centrifugation at $2500 \mathrm{~g}$ for $5 \mathrm{~min}$ within 12 hours of collection. The magnetic beads of these CLIA assays are coated with two antigens of SARS-CoV-2 (nucleocapsid protein (N protein) and spike protein (S protein)). SARS-CoV$2 \mathrm{IgM} / \mathrm{IgG}$ titers (in arbitrary units, AU/ml) were calculated automatically by the CLIA analyzer based on relative light units (RLU), and the viral antibody titer was positively associated with RLU. The cutoff values for positive SARS-CoV-2 IgM and IgG are both $10 \mathrm{AU} / \mathrm{ml}$.

2.3. SARS-CoV-2 Nucleic Acid Test. RT-PCR was used to detect open reading frame $1 \mathrm{ab}(\mathrm{ORF} 1 \mathrm{ab})$ and nucleocapsid protein $(\mathrm{N})$ in the SARS-CoV-2 genome. CT value interpretation of test results is based on the instruction from the manufacturer. Confirmation of positive COVID-19 is based on at least one target-specific RT-PCR-positive result of ORF1ab and $\mathrm{N}$ genes of SARS-CoV-2 in the same specimen.

2.4. Data Analysis. Statistical analysis was performed using SPSS 19.0 statistical software (IBM SPSS, Chicago, IL, USA). The kappa coefficient was calculated. Kappa $\geq 0.75$ indicates good consistency, $0.75 \geq$ kappa $>0.4$ for medium consistency, and kappa $<0.4$ for poor consistency.

The specificity and sensitivity of the CLIA test kits were calculated according to the following equations:

$$
\begin{aligned}
& \text { sensitivity }(\%)=100 \% \times\left[\frac{\text { true positive }}{(\text { true positive }+ \text { false negative })}\right], \\
& \text { specificity }(\%)=100 \% \times\left[\frac{\text { true negative }}{(\text { true negative }+ \text { false positive })}\right] .
\end{aligned}
$$

\section{Results}

3.1. Specificity of SARS-CoV-2 IgG/IgM Antibody Test. Samples from both NAT-negative patients (suspected group, 9 subjects) and other diseases' population (control group, 101 subjects) were used to assess the clinical specificity of the assay (Table 1). Among the 101 patients in the control group, 100 patients were negative for SARS-CoV-2 IgM antibody, with a clinical specificity of $99.01 \%$ (100/101). 97 patients were negative for SARS-CoV-2 IgG antibody, with a clinical specificity of $96.04 \%$ (97/101). In the suspected group, all 9 patients had negative antibody test results. The false-positive results of SARS-CoV-2 IgM and IgG antibodies may be caused by auto-antibodies, heterophilic antibodies, and other factors.

\subsection{Detection Sensitivity of SARS-CoV-2 IgG/IgM Antibody} Test. Samples from 68 SARS-CoV-2-infected patients (confirmed with RT-PCR) were used to evaluate the clinical sensitivity of the assays (Table 2). We analyzed the clinical sensitivity on both SARS-CoV-2 IgM and IgG antibodies at three time periods, before 7 days, 7-14 days, and after 14 days since the onset of the symptoms. During these time periods, SARS-CoV-2 IgM demonstrated a clinical sensitivity of $75.00 \%, 88.00 \%$, and $93.55 \%$, respectively, and SARS-CoV-2 IgG demonstrated 83.33\%, $100.00 \%$, and $100.00 \%$, respectively. The total clinical sensitivity of SARSCoV-2 IgM and IgG to SARS-CoV-2 infection was $88.24 \%$ $(60 / 68)$ and $97.06 \%(66 / 68)$, respectively.

3.3. Comparison of SARS-CoV-2 Antibody Test and SARSCoV-2 Nucleic Acid Test (NAT). The comparison between SARS-CoV-2 IgM/IgG antibody test and NAT of 178 patients is shown in Table 3. The positive predictive value of SARSCoV-2 IgM/IgG antibody detection was $93.06 \%$ (67/72), and the negative predictive value was $99.06 \%(105 / 106)$. The positive predictive value of the NAT for SARS-CoV-2 was $100 \%(68 / 68)$, and the negative predictive value was $96.36 \%$ $(106 / 110)$.

\section{Discussion}

Novel coronavrius disease is caused by severe acute respiratory syndrome coronavirus 2 (SARS-CoV-2) [7]. SARSCoV-2 belongs to the subfamily of Coronavirinae (named Betacoronavirus), and its genome is a single-stranded positive-sense RNA [8]. Different from MERS-CoV and SARSCoV, SARS-CoV-2 is the seventh member of the family of coronaviruses that infect humans [9]. The disease (COVID19) has been rapidly spreading for the past five months, and now it has been found in more than 215 countries. By June 19, 2020, over 8,300,000 COVID-19-confirmed patients were reported, with $>450,000$ deaths. Currently, the SARSCoV-2 NAT is the routine confirmation test for the clinical diagnosis of COVID-19 [2]. However, not all clinical COVID-19 patients might have positive results from SARSCoV-2 NAT. The reasons of false-negative NAT results in COVID-19 include collection and storage of the sample, the 
TABle 1: Clinical specificity of SARS-CoV-2 IgM and SARS-CoV-2 IgG.

\begin{tabular}{lccccccccc}
\hline & \multirow{2}{*}{ Number of samples } & \multicolumn{4}{c}{ SARS-CoV-2 IgM } & \multicolumn{3}{c}{ SARS-CoV-2 IgG } \\
& & $\mathrm{N}$ & $\mathrm{P}$ & Clin Spe (\%) & 95\% CI & $\mathrm{N}$ & $\mathrm{P}$ & Clin Spe (\%) & 95\% CI \\
\hline Suspected group & 9 & 9 & 0 & 100.00 & $(70.1 \%, 100.0 \%)$ & 9 & 0 & 100.00 & $(70.1 \%, 100.0 \%)$ \\
Control group & 101 & 100 & 1 & 99.01 & $(94.6 \%, 99.8 \%)$ & 97 & 4 & 96.04 & $(90.3 \%, 98.4 \%)$ \\
\hline
\end{tabular}

N: negative; P: positive; Clin Sep: clinical specificity.

TABLE 2: Clinical sensitivity of SARS-CoV-2 IgM and SARS-CoV-2 IgG.

\begin{tabular}{|c|c|c|c|c|c|c|c|c|c|}
\hline \multirow{2}{*}{ Days } & \multirow{2}{*}{ Number of samples } & \multicolumn{4}{|c|}{ SRAS-CoV-2 IgM } & \multicolumn{4}{|c|}{ SRAS-CoV-2 IgG } \\
\hline & & $\mathrm{N}$ & $\mathrm{P}$ & Clin Sen (\%) & $95 \% \mathrm{CI}$ & $\mathrm{N}$ & $\mathrm{P}$ & Clin Sen (\%) & $95 \% \mathrm{CI}$ \\
\hline$<7$ days & 12 & 3 & 9 & 75.00 & $(46.8 \%, 91.1 \%)$ & 2 & 10 & 83.33 & $(55.2 \%, 95.3 \%)$ \\
\hline 7-14 days & 25 & 3 & 22 & 88.00 & $(70.0 \%, 95.8 \%)$ & 0 & 25 & 100.00 & $(86.7 \%, 100.0 \%)$ \\
\hline$>14$ days & 31 & 2 & 29 & 93.55 & $(79.3 \%, 98.2 \%)$ & 0 & 31 & 100.00 & $(89.0 \%, 100.0 \%)$ \\
\hline Total & 68 & 8 & 60 & 88.24 & $(78.5 \%, 93.9 \%)$ & 2 & 66 & 97.06 & $(89.9 \%, 99.2 \%)$ \\
\hline
\end{tabular}

Days: days since the onset of symptoms; N: negative; P: positive; Clin Sen: clinical sensitivity.

TABle 3: Comparison of SARS-CoV-2 IgM/IgG antibody detection and SARS-CoV-2 nucleic acid detection.

\begin{tabular}{|c|c|c|c|c|c|c|}
\hline \multicolumn{7}{|c|}{ IgM/IgG antibody } \\
\hline & & Positive & Negative & Total & $\begin{array}{c}\text { Positive predictive value } \\
\text { of NAT (\%) }\end{array}$ & $\begin{array}{c}\text { Negative predictive value } \\
\text { of NAT }(\%)\end{array}$ \\
\hline \multirow{3}{*}{ Nucleic acid } & Positive & 67 & 1 & 68 & 100.00 & \multirow{3}{*}{96.36} \\
\hline & Negative & 5 & 105 & 110 & & \\
\hline & Total & 72 & 106 & 178 & & \\
\hline Positive predictive value of antibody test & & & & & 93.06 & \\
\hline Negative predictive value of antibody test & & & & & & 99.06 \\
\hline
\end{tabular}

condition of the NAT laboratory, and the quality of the test kit [10]. Therefore, nucleic acid detection, CT imaging, blood routine examination, and other methods can be used together for the comprehensive diagnosis of COVID-19.

Since February 2020, several SARS-COV-2 IgM and IgG antibody immunoassay kits have been developed in China. Antibody detection is a new detection method for SARSCoV-2, so the clinical specificity and sensitivity of such tests must be carefully validated [11]. Our study demonstrated that the SARS-CoV-2 IgM and IgG CLIA kits (YHLO Biotech, Shenzhen, China) had a high clinical specificity, reaching $99.01 \%$ and $96.04 \%$, respectively. Therefore, SARSCoV-2 IgM and IgG antibody detection reagents have high clinical specificity and can meet the screening and diagnosis requirements of SARS-CoV-2.

In COVID-19 cases, the clinical sensitivity of SARSCoV-2 IgM detection was $88.23 \%$, while the clinical sensitivity of SARS-CoV-2 IgG detection was $97.06 \%$. The CLIA system can simultaneously detect 150-300 clinical samples, which is a good tool for screening and diagnosis of the novel coronavirus pneumonia caused by SARS-CoV-2. Our study results showed that the combined detection of SARS-CoV-2 IgM and IgG antibodies is an effective tool to improve the diagnostic sensitivity and specificity and reduce the chance of false-negative NAT results. We demonstrate that the antibody detection can be used as one of the effective methods of COVID-19 clinical detection.

In the NAT-confirmed group, serum from 68 COVID19 cases was tested for SARS-CoV-2 IgM and IgG. SARS-
CoV-2 IgM antibodies can be detected in $75.00 \%$ of patients before 7 days since the onset of the symptoms, and the positive rate reached to $88.00 \%$ on the period of $7-14$ days and then increased to $93.55 \%$ after 14 days. The positive rate of SARS-CoV-2 IgG was $83.33 \%$ before 7 days since the onset of symptoms and reached to $100.00 \%$ on $7-14$ days and remained $100 \%$ after 14 days. In general, the immune response to infection by pathogenic microorganisms is first expressed as an increase in the IgM antibody titer and then a rapid decrease until it disappears, while the $\operatorname{IgG}$ antibody titer normally increased in the middle and late stages of the infection, and it can be positive for a long time even after recovery. According to the results of this study, the positive rate of IgM in SARS-CoV-2-infected patients is lower than that of IgG because most of the infected patients were in the middle stage of infection or in the recovery stage. Interestingly, we have observed a phenomenon that SARS-CoV-2 IgM and IgG antibodies developed almost simultaneously, and this observation is consistent with some recent studies [12]. Further studies are needed to verify this phenomenon in the diagnosis and prognosis of COVID-19.

We found false-negative results for IgM/IgG in the NAT group. There might be three reasons: first of all, false-negative results may be due to low antibody titer. When IgM and IgG titers are below the detection limit, the test result might be negative. Secondly, the difference in individual immune response and antibody production could be another reason for the false-negative results in COVID-19 patients. The last reason might be that IgM antibody might decrease or even 
disappear after 15 days. In each individual case, it is difficult to know exactly when or how long the patient has been really infected, and someone might have IgM titer below the detection limit and not detectable. In the joint detection of SARS-CoV-2 IgM and IgG, there was only one negative patient (male, 77 years old) who had respiratory failure, chronic obstructive pulmonary disease, coronary atherosclerosis, acute myocardial infarction, and heart failure with SARS-CoV-2 infection. In the control group, 5 cases were positive for antibody detection ( 1 case for IgM and 4 cases for $\operatorname{IgG})$. The results suggested that the patients who had some other diseases, including tumors, leukemia, diabetes, hypertension, coronary atherosclerosis, bronchitis, or lung infections, might be more susceptible to be infected by SARS-CoV-2 and led to positive antibody detection. Also, there might be false-negative nucleic acid or recovered/mild/ asymptomatic patients with SARS-CoV-2. In addition, it is well known that the positive and negative predictive values are not only intrinsic to the test but also depend on the prevalence [13]. Therefore, predictive values shown in Table 3 are only valid for the sample used in the study, and they are not valid for other facilities or for the general population. All of these cases will provide valuable reference for the follow-up study and clinical diagnosis of COVID-19.

Our study also has some limitations. For example, we did not investigate the cross-reaction with other pathogens (e.g., hCoV-NL-63 or others), MERS-CoV, SARS-CoV, and some auto-antibodies that could cause interference for immunoassay. Also, we did not perform dynamic monitoring of the change of antibody titer for in-depth study.

\section{Conclusions}

Overall, testing SARS-CoV-2 IgG and IgM by the CLIA method is convenient for sampling, and it has high efficiency. The results of this study indicated that combined detection of serum IgM and IgG antibodies to SARS-CoV-2 had better sensitivity and specificity compared with single IgM or IgG antibody testing. Therefore, the serological test results can be used as an effective diagnostic tool for SARS$\mathrm{CoV}-2$ infection. It can also be used as an efficient supplement of RNA detection for confirmation of SARS-CoV-2 infection in clinics, hospitals, and accredited scientific laboratories.

\section{Abbreviations}

$\begin{array}{ll}\text { SARS-CoV-2: } & \begin{array}{l}\text { Severe acute respiratory syndrome } \\ \text { coronavirus } 2\end{array} \\ \text { NAT: } & \text { Nucleic acid test } \\ \text { CLIA: } & \text { Chemiluminescence immunoassay } \\ \text { IgM: } & \text { Immunoglobulin M } \\ \text { IgG: } & \text { Immunoglobulin G. }\end{array}$

\section{Data Availability}

The datasets used and/or analyzed during the current study are available from the corresponding author upon reasonable request.

\section{Disclosure}

All the SARS-CoV-2 IgG and IgM CLIA kits used in this study were kindly supplied by the manufacturer, namely, YHLO Biotech (Shenzhen, China).

\section{Conflicts of Interest}

The authors declare that they have no conflicts of interest.

\section{Authors' Contributions}

Fang $\mathrm{Hu}$ analyzed the data, drafted the article, and contributed to study design. Xiaoling Shang and Changliang Zhang contributed to data gathering. Meizhou Chen contributed to study design, editing, and revising the paper. All authors read and approved the final manuscript.

\section{References}

[1] D. S. Hui, E. I. Azhar, T. A. Madani et al., "The continuing 2019-nCoV epidemic threat of novel coronaviruses to global health-the latest 2019 novel coronavirus outbreak in Wuhan, China," International Journal of Infectious Diseases, vol. 91, pp. 264-266, 2020.

[2] V. M. Corman, O. Landt, M. Kaiser et al., "Detection of 2019 novel coronavirus (2019-nCoV) by real-time RT-PCR," Euro Surveillance, vol. 25, no. 3, Article ID 2000045, 2020.

[3] J. Li, G. Ye, L. Chen, J. Wang, and Y. Li, "Analysis of falsenegative results for 2019 novel coronavirus nucleic acid test and related countermeasures," Chinese Journal of Laboratory Medicine, vol. 43, Article ID E006, 2020.

[4] Y. Jin, M. Wang, Z. Zuo et al., "Diagnostic value and dynamic variance of serum antibody in coronavirus disease 2019," International Journal of Infectious Diseases, vol. 94, pp. 49-52, 2020.

[5] J. Qu, C. Wu, X. Li et al., "Profile of IgG and IgM antibodies against severe acute respiratory syndrome coronavirus 2 (SARS-CoV-2)," Clinical Infectious Diseases, Article ID ciaa489, 2020.

[6] D. Chen, Y. Zhang, Y. Xu et al., "Comparison of chemiluminescence immunoassay, enzyme-linked immunosorbent assay and passive agglutination for diagnosis of mycoplasma pneumoniae infection," Therapeutics and Clinical Risk Management, vol. 14, pp. 1091-1097, 2018.

[7] C. Huang, Y. Wang, X. Li et al., "Clinical features of patients infected with 2019 novel coronavirus in Wuhan, China," The Lancet, vol. 395, no. 10223, pp. 497-506, 2020.

[8] Y. Chen, Q. Liu, and D. Guo, "Emerging coronaviruses: genome structure, replication, and pathogenesis," Journal of Medical Virology, vol. 92, no. 4, pp. 418-423, 2020.

[9] N. Zhu, D. Zhang, W. Wang et al., "A novel coronavirus from patients with pneumonia in China, 2019," New England Journal of Medicine, vol. 382, no. 8, pp. 727-733, 2020.

[10] X. Mo, W. Qin, Q. Fu, and M. Guan, "Understanding the influence factors in viral nucleic acid test of 2019 novel coronavirus (2019-nCoV)," Chinese Journal of Laboratory Medicine, vol. 43, Article ID E002, 2020.

[11] J. Zhao, Q. Yuan, H. Wang et al., "Antibody responses to SARS-CoV-2 in patients of novel coronavirus disease 2019," Clinical Infectious Diseases, Article ID ciaa344, 2020. 
[12] Q. X. Long, B. Z. Liu, H. J. Deng et al., "Antibody responses to SARS-CoV-2 in patients with COVID-19," Nature Medicine, vol. 20, no. 6, pp. 845-848, 2020.

[13] D. G. Altman and J. M. Bland, "Statistics notes: diagnostic tests 2: predictive values,” BMJ, vol. 309, no. 6947, p. 102, 1994. 